\title{
Fotosíntesis en tres poblaciones altitudinales de la planta andina Espeletia schultzii (Compositae)
}

\author{
Marisol Castrillo \\ Depto. Biología de Organismos, Universidad Simón Bolívar. Apdo. Postal 89000, Caracas 1080ª Venezuela; \\ mcastr@usb.ve
}

\author{
Recibido 04-VIII-2003. Corregido 08-IX-2005. Aceptado 12-V-2006.
}

\begin{abstract}
Photosynthesis in three altitudinal populations of the Andean plant Espeletia schultzii (Compositae). Photosynthesis was compared in three altitudinal populations of Espeletia schultzii: 3 100, 3550 and 4200 masl. The measured parameters were Rubisco activity (EC 4.1.139), chlorophyll, soluble protein and soluble sugars contents, and specific leaf area (SLA). The $4200 \mathrm{~m}$ population had a higher Rubisco activity (at $4{ }^{\circ} \mathrm{C}$ ) followed by those at $3550 \mathrm{~m}$ and $3100 \mathrm{~m}$. There were no significant differences between populations at $3100 \mathrm{~m}$ and $3550 \mathrm{~m}$ (ANOVA), but their activities were different from those of the $4200 \mathrm{~m}$ population. Chlorophyll a content decreased slightly with elevation, while chlorophyll b was constant; therefore, the Ra/b ratio decreased with elevation, but not significantly. The leaf soluble sugars content increased along the altitudinal gradient. Leaf protein content did not differ. The SLA decreased with altitude. The increase in Rubisco activity might reflect higher enzyme activation and not higher enzyme protein. The increase in soluble sugars is probably associated to Rubisco activity. Three out of the five measured parameters revealed differences with altitude, suggesting a compromise between a higher metabolic activity and a smaller cellular volume. Rev. Biol. Trop. 54 (4): 1143-1149. Epub 2006 Dec. 15.
\end{abstract}

Key words: chlorophyll, Espeletia, paramo, rubisco, soluble proteins, soluble sugars, specific leaf area.

El páramo es un bioma neotropical de alta montaña; Meinzer et al. (1985) lo describen como una zona caracterizada por elevada radiación solar y baja energía térmica; los suelos son poco profundos con escaso contenido de nutrientes (Vareschi 1970, Baruch 1979, Monasterio 1980). En este clima tropical andino, las plantas están expuestas a cambios climáticos diarios en lugar de estacionales; igualmente pueden estar sometidas a frecuentes episodios de congelamiento (Monasterio 1980). A lo largo del gradiente altitudinal, la temperatura del aire y la precipitación disminuyen con la elevación, mientras la velocidad del viento, la radiación total y ultravioleta y la tasa de evaporación aumentan a mayor elevación. Goldstein et al. (1985) registraron valores para la temperatura mínima promedio y la precipitación anual de $6.23{ }^{\circ} \mathrm{C}$ (estimada) y $1213 \mathrm{~mm}$ para $3100 \mathrm{~m}$ de altitud, $4.77{ }^{\circ} \mathrm{C}$ y $969 \mathrm{~mm}$ para $3550 \mathrm{~m}$, y $-0.02{ }^{\circ} \mathrm{C}$ y $789 \mathrm{~mm}$ para $4200 \mathrm{~m}$.

La vegetación dominante está constituida por rosetas gigantes, arbustos y gramíneas. (Baruch 1979, 1984). Frailejón es el nombre común de las especies del género Espeletia. La especie Espeletia schultzii es una planta roseta que crece en los Andes venezolanos (Sierra Nevada de Mérida $8^{\circ} 5^{\prime} \mathrm{N}, 70^{\circ} 45^{\prime} \mathrm{W}$; Estado Mérida, Venezuela); sus poblaciones están distribuidas a través del ámbito altitudinal de los páramos desde 2600 hasta 4200 msnm y están diferenciadas por el grado de resistencia a la sequía, siendo las más resistentes aquéllas que se encuentran a mayor elevación; también es característico que la pubescencia y el grosor de las hojas aumenten con la elevación (Baruch 1979, Meinzer et al. 1985). El congelamiento 
de las hojas en condiciones naturales no ha sido informado para las especies de Espeletia y el punto de sobre-enfriamiento decrece a lo largo del gradiente altitudinal entre -6.5 y $-10.5{ }^{\circ} \mathrm{C}$ (Goldstein et al. 1985). El sobre-enfriamiento no altera el estado hídrico y los procesos metabólicos continúan a temperaturas por debajo de $0{ }^{\circ} \mathrm{C}$, aunque a tasas reducidas.

Diversos estudios del género Espeletia han sido publicados, tales como demografía comparativa entre dos especies E. timotensis y $E$ spicata (Silva et al. 2000); filogenia molecular del complejo Espeletia (Asteraceae) (Rauscher 2002); identificación de aceites naturales (Khouri et al. 2000, Usubillaga et al. 2001); evaluación fitoquímica y actividad antibacterial (De los Ríos et al.1999).

Con relación a los efectos elevacionales sobre la fotosíntesis, los resultados pueden ser contradictorios. Machler y Nosberger (1977) trabajando con ecotipos de Trifolium repens encontraron que aquellos ubicados a mayor altitud requieren menores temperaturas para fotosintetizar; mientras que Kemp y William (1977) no observaron diferencias en fotosíntesis neta, fotorrespiración y transpiración en poblaciones altitudinales de Taraxacum officinale. En tres poblaciones de E. schultzii, 3 100, 3550 y $4200 \mathrm{msnm}$, la tasa de fijación de $\mathrm{CO}_{2}$ aumenta con la temperatura, los valores de Vmax para la actividad Rubisco son mayores en la población ubicada a mayor altitud; la $\mathrm{Km}\left(\mathrm{CO}_{2}\right)$ incrementa con la temperatura y es similar entre las poblaciones (Castrillo 1995). El contenido foliar de carbohidratos totales no estructurales, expresados sobre peso seco, no es significativamente diferente entre las poblaciones (Castrillo y Simoes 1997).

El objetivo del presente trabajo interpretar el efecto de la altitud en el metabolismo fotosintético de E. schultzii, continuando con los estudios que, sobre esta especie típica de los Andes Venezolanos, hemos venido realizando. Para ello se analizaron los siguientes parámetros fotosintéticos: actividad Rubisco (Ribulosa 1, 5-bis-fosfato carboxilasa EC 4.1.1.39); contenidos de clorofila, azúcares solubles totales y proteínas solubles y área foliar específica
(AFE) en tres poblaciones de la especie ubicadas a 3 100, 3550 y 4200 msnm.

\section{MATERIALES Y MÉTODOS}

Se tomaron diez plantas de cada población. De cada una de ellas fueron recolectadas hojas jóvenes recién expandidas, cercanas al ápice.

La población de $3100 \mathrm{msnm}$ fue obtenida en las cercanías del Hotel Los Frailes; la de $3550 \mathrm{~m}$, en las adyacencias de la Laguna de Mucubají, y la de $4200 \mathrm{~m}$, en la vía hacia el caserío de Piñango. Estas localidades están ubicadas en el Estado Mérida $\left(8^{\circ} 5^{\prime} \mathrm{N}, 70^{\circ} 45^{\prime}\right.$ W). El muestreo fue realizado entre las 11:00 y 15:00 h. Las hojas fueron almacenadas en bolsas plásticas mantenidas en hielo en envases portátiles y transportadas de inmediato al laboratorio por vía aérea (aproximadamente $3 \mathrm{~h}$ ).

El peso fresco y el seco (luego de mantener las hojas en una estufa por $48 \mathrm{~h}$ a $80^{\circ} \mathrm{C}$ ) fueron determinados en una balanza analítica. El área foliar fue medida con un planímetro (LasikoEst 1929, Los Angeles Scientific Instrument Co., Inc., Los Angeles, California, EEUU).

Las hojas fueron mantenidas en nitrógeno líquido hasta ser utilizadas para los análisis (de una a ocho semanas después de colectadas). Diez "réplicas" provenientes de diez plantas individuales fueron usadas en tres repeticiones.

Se siguió la metodología utilizada por Castrillo (1995) para la extracción (en frío) y ensayo de la actividad Rubisco. El medio de extracción contenía: $0.2 \mathrm{M}$ Tricina- $\mathrm{NaOH}, \mathrm{pH}$ 8.2, $0.02 \mathrm{M} \mathrm{MgCl}_{2}, 0.01 \mathrm{M} \mathrm{NaHCO}_{3}, 0.0002 \mathrm{M}$ EDTA (ácido etilendiamintetracético), $0.02 \mathrm{M}$ 2-Mercaptoetanol y $20 \mathrm{~g} / 1$ de polivinilpolipirrolidona (PVPP). La vena central de las hojas fue removida, el mesófilo cortado en segmentos de $0.25 \mathrm{~cm}^{2}$ y homogeneizado en una relación 1:10 (p/v). La mezcla fue homogeneizada en una licuadora por 1 min a baja y 2 min a más alta velocidad. El homogenato fue filtrado a través de cuatro capas de muselina. El filtrado fue desalado por centrifugación a través de una columna Sephadex G-25. La actividad 
Rubisco fue medida a la temperatura de $4{ }^{\circ} \mathrm{C}$, que representa el promedio de los valores de temperatura registrados en las tres altitudes en las tres poblaciones estudiadas. El medio de ensayo contenía, en un volumen final de $200 \mu \mathrm{l}, 0.15$ M Tricina-NaOH, pH 8.2, 0.02 $\mathrm{M} \mathrm{MgCl}, 0.04 \mathrm{M}$ 2-Mercaptoetanol, 0.002M RuBP, $0.01 \mathrm{M} \mathrm{NaH}{ }^{14} \mathrm{CO}_{3}(3.7 \mathrm{MBq} / \mathrm{mmol})$; la reacción comenzó con la adición de $30 \mu \mathrm{l}$ del extracto foliar. El tiempo de reacción fue $1 \mathrm{~min}$, la reacción fue detenida con $200 \mu \mathrm{l}$ de ácido acético al $25 \%$. La radioactividad incorporada en ácidos estables fue medida en un contador de centelleo líquido (LKB Wallac 1217 Rackbeta). Los ensayos fueron realizados por duplicado.

En el extracto foliar utilizado para el ensayo enzimático, se determinaron los contenidos de clorofila, azúcares y proteínas. El de clorofila en acetona al $80 \%$, siguiendo el método de Bruinsma (1963). El de azúcares solubles, utilizando el método de antrona $(150 \mathrm{mg}$ antrona/100 $\mathrm{ml}$ de ácido sulfúrico al $75 \%$ ); se incubó por $20 \mathrm{~min}$ a $40{ }^{\circ} \mathrm{C}$, y se midió la absorbancia a $620 \mathrm{~nm}$; fue utilizada una curva de calibración con soluciones de sacarosa (Van Handel 1968). El contenido de proteínas fue medido por el método de Bradford (1976) utilizando albúmina sérica de bovino para la curva de calibración.

Un análisis de varianza (ANOVA)fue utilizado para el tratamiento estadístico de los resultados (Sokal y Rohlf 1969).

\section{RESULTADOS}

En el Cuadro 1 se presentan los valores de la actividad Rubisco expresados en $\mu \mathrm{mol}^{14} \mathrm{CO}_{2} \mathrm{~m}^{-2} \mathrm{~min}^{-1}$, medida a $4{ }^{\circ} \mathrm{C}$ en las tres poblaciones altitudinales estudiadas. $\mathrm{La}$ población ubicada a 4200 m presentó mayor actividad seguida por las poblaciones a 3550 y a $3100 \mathrm{~m} \mathrm{El}$ análisis de varianza reveló que no existen diferencias significativas entre las poblaciones a 3100 y $3550 \mathrm{~m}$ pero sí, entre estas dos y aquélla que se encuentra a $4200 \mathrm{~m}$ $(\mathrm{F}=10.233, \mathrm{gl}=2, \mathrm{p}=0.0016)$.
CUADRO 1

Actividad Rubisco $\left(\mathrm{\mu mol}^{14} \mathrm{CO}_{2} \mathrm{~m}^{-2} \mathrm{~min}^{-1}\right)$ en las tres
poblaciones de E. schultzii

TABLE 1

Rubisco activity $\left(\mathrm{\mu mol}^{14} \mathrm{CO}_{2} \mathrm{~m}^{-2} \mathrm{~min}^{-1}\right)$ in three altitudinal populations of E. schultzii assayed at $4{ }^{\circ} \mathrm{C}$ (recorded temperature average from the three altitudes)

$\begin{array}{cccc}\text { Población } & 3100 \mathrm{~m} & 3550 \mathrm{~m} & 4200 \mathrm{~m} \\ \begin{array}{l}\text { Actividad Rubisco } \\ \left(\mu \mathrm{mol}^{14} \mathrm{CO}_{2} \mathrm{~m}^{-2} \mathrm{~min}^{-1}\right)\end{array} & 243 \pm 93 & 355 \pm 82 & 485 \pm 99\end{array}$

Los valores representan la media \pm desviación estándard. The values are the means \pm standard deviation.

En la Fig. 1 se observa que los contenidos de clorofila a muestran una leve tendencia a la disminución con la altura, mientras que la clorofila $b$ se mantiene constante, razón por la cual la relación a/b $(\mathrm{R}$ a/b) disminuye con la elevación. No obstante, el ANOVA revela que las diferencias entre las poblaciones no son significativas $(\mathrm{F}=1.690, \mathrm{gl}=2, \mathrm{p}=0.2005)$.

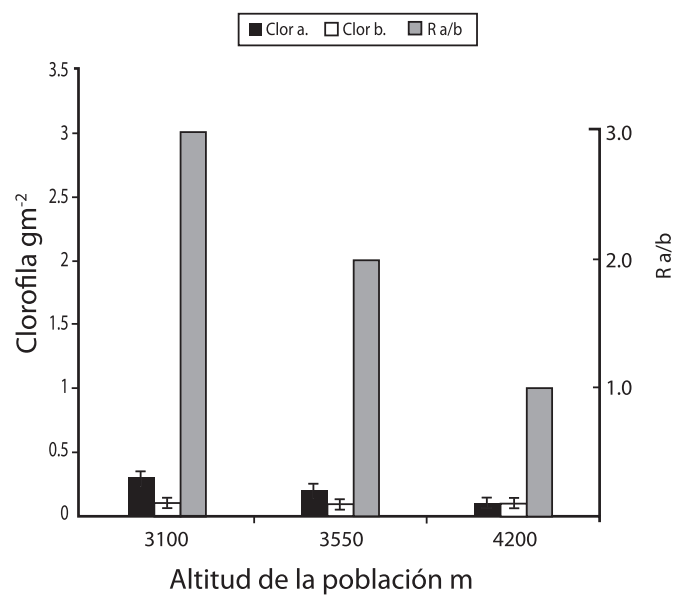

Fig. 1. Contenido de clorofilas a y $\mathrm{b}\left(\mathrm{g} \cdot \mathrm{m}^{-2}\right)$ y Relación $\mathrm{a} / \mathrm{b}(\mathrm{R} \mathrm{a} / \mathrm{b})$ en tres poblaciones altitudinales de E. schultzii. Los valores representan las medias de diez repeticiones \pm desviación estándar.

Fig. 1. Chlorophyll a and b contents $\left(\mathrm{g} \cdot \mathrm{m}^{-2}\right)$ and $\mathrm{a} / \mathrm{b}$ ratio $(\mathrm{Ra} / \mathrm{b})$ in three altitudinal populations of E. schultzii. The values are the means of ten replicates \pm standard deviation. 
El contenido de azúcares $\left(\mathrm{gm}^{-2}\right)$ es mostrado en la Fig. 2; se observa un aumento con la elevación; el análisis de varianza revela diferencias significativas entre las tres poblaciones $(\mathrm{F}=20.314, \mathrm{gl}=2, \mathrm{p}=0.0001)$.

El contenido de proteínas se presenta en la Fig. 3; aquí el valor mayor lo presenta la población ubicada a $4200 \mathrm{~m}$, y el menor contenido se presenta a $3550 \mathrm{~m}$; la población ubicada a $3100 \mathrm{~m}$ presenta un valor intermedio. El análisis de varianza, sin embargo, mostró que no hay diferencias significativas entre las poblaciones $(\mathrm{F}=1.770, \mathrm{gl}=2, \mathrm{p}=0.2009)$.

La Fig. 4 muestra los valores obtenidos para el área foliar específica (AFE) expresados en $\mathrm{m}^{2} 10^{-2} \mathrm{~g}^{-1} \mathrm{PS}$; el análisis de varianza demuestra que existen diferencias significativas entre las poblaciones $(\mathrm{F}=32.957, \mathrm{gl}=2$, $\mathrm{p}<0.0001$ ).

\section{DISCUSIÓN}

Se evidencia una mayor actividad de Rubisco en la población ubicada a mayor elevación. Aumentos de la tasa de fijación de $\mathrm{CO}_{2}$ y de Vmax en Rubisco con la altitud en E. schultzii fueron encontrados por Castrillo (1995), lo que concuerda con el incremento en actividad observado a $4200 \mathrm{~m}$. Este aumento de la actividad Rubisco con la altitud pudiera deberse a mayor contenido de proteínas y/o mayor activación de esta enzima.

Al igual que lo obtenido en este trabajo, en Taraxacum officinale se mostró aumento de la actividad Rubisco con la altitud (Oulton et al. 1979). Por otra parte, en un estudio sobre poblaciones de E. schultzii, se encontró que las plantas provenientes de localizaciones a mayor altura y sometidas a aclimatación en frío, mostraron altas tasas de asimilación, mientras que aquéllas provenientes de sitios de menor altitud, presentaron elevadas tasas a mayores temperaturas de aclimatación (Baruch 1979). Asimismo, Rada et al. (1992) comunicaron una disminución del óptimo de temperatura foliar para la fotosíntesis acoplada a una disminución de la temperatura del aire a lo largo

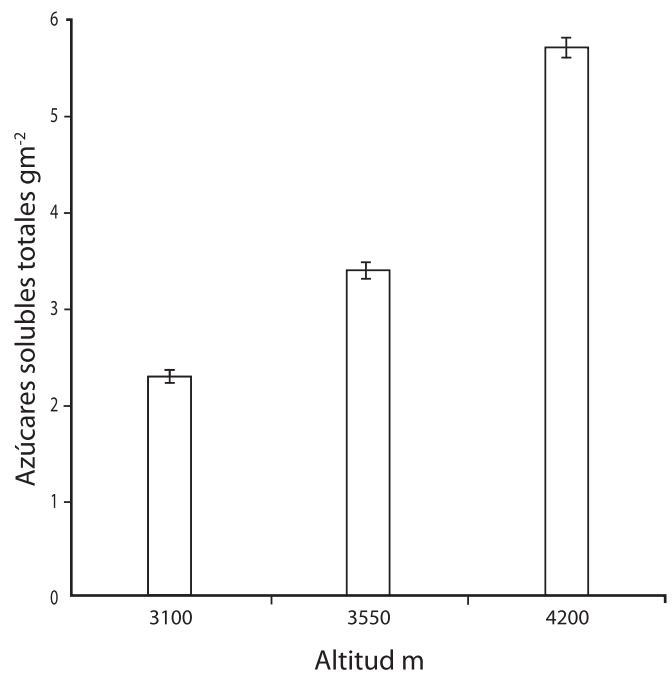

Fig. 2. Contenido de azúcares solubles $\left(\mathrm{g} \cdot \mathrm{m}^{-2}\right)$ y Relación $\mathrm{a} / \mathrm{b}(\mathrm{R} \mathrm{a} / \mathrm{b})$ en tres poblaciones altitudinales de $E$. schultzii. Los valores representan las medias de diez repeticiones 土desviación estándar.

Fig. 2. Soluble sugars content contents $\left(\mathrm{g} \cdot \mathrm{m}^{-2}\right)$ in three altitudinal populations of E. schultzii. The values are the means of ten replicates \pm standard deviation.

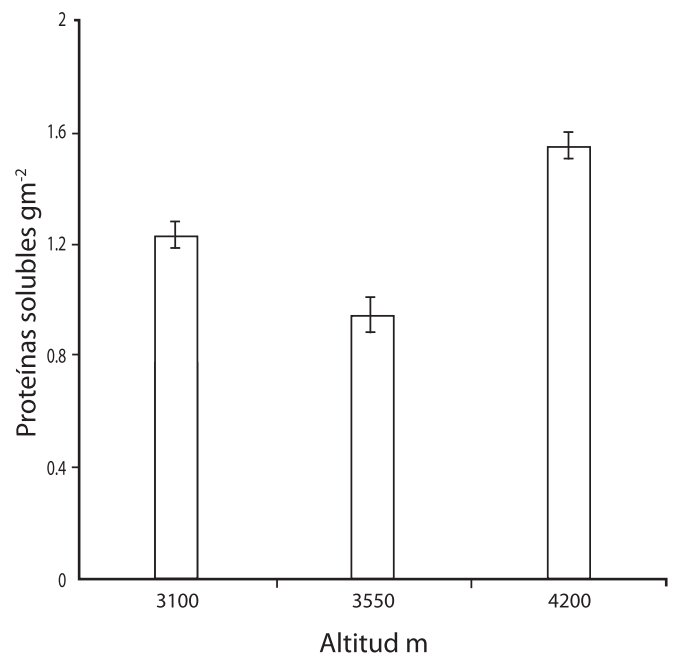

Fig. 3. Contenido de proteínas solubles $\left(\mathrm{g} \cdot \mathrm{m}^{-2}\right)$ en tres poblaciones altitudinales de E. schultzii. Los valores representan las medias de diez repeticiones \pm desviación estándar.

Fig. 3. Soluble protein content $\left(\mathrm{g} \cdot \mathrm{m}^{-2}\right)$ in three altitudinal populations of E. schultzii. The values are the means of ten replicates \pm standard deviation. 


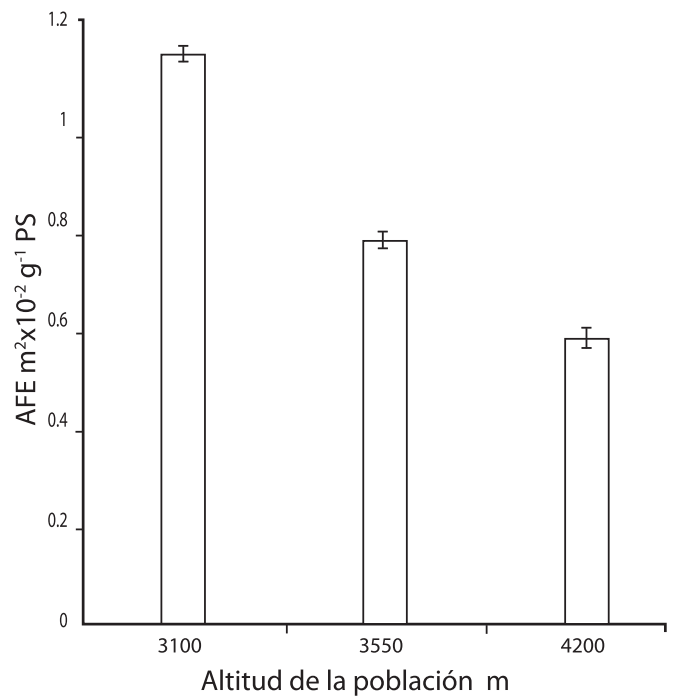

Fig. 4. Área Foliar Específica (AFE) $\left(\mathrm{m}^{2} 10^{-2} \mathrm{~g}^{-1} \mathrm{PS}\right)$ en tres poblaciones altitudinales de E. schultzii. Los valores representan las medias de diez repeticiones \pm desviación estándar.

Fig. 4. Specific leaf area $\left(\mathrm{m}^{2} 10^{-2} \mathrm{~g}^{-1} \mathrm{PS}\right)$ in three altitudinal populations of $E$. schultzii. The values are the means of ten replicates \pm standard deviation.

de un gradiente para dos poblaciones de $E$. schultzii desde 2950 hasta $4000 \mathrm{~m}$. Se ha informado también, que existen diferencias significativas en la tasa de asimilación de $\mathrm{CO}_{2}$ en tres poblaciones altitudinales de E. schultzii, entre la estación seca y la de lluvias y una diferenciación en este intercambio gaseoso con la altitud. La disminución en este parámetro con el gradiente altitudinal es explicada por factores como pubescencia, baja conductancia foliar y menor contenido de nitrógeno en el suelo (Rada et al. 1998).

La diferencia no significativa en el contenido de clorofila, entre las poblaciones altitudinales, puede deberse al efecto protector de las pubescencia, que incrementa con la elevación, (demostrado por Baruch 1979, Meinzer et al. 1985, Rada et al. 1998 y observado en nuestro laboratorio) previniendo así a la clorofila de la fotoxidación. En el análisis del contenido de clorofila, no se obtuvo una disminución significativa con la altitud, tal y como se ha informado en poblaciones alpinas (Tranquilini
1979); el efecto fotooxidativo de la radiación sobre la clorofila puede ser atenuado por el efecto protector que pudiera ejercer el incremento en la pubescencia con la elevación. Es así que una adaptación morfológica protege del efecto adverso de excesiva radiación asociado con la elevación.

Se observa un notable aumento en el contenido de azúcares con la elevación; este hecho es atribuible a la mayor actividad carboxilasa de la Rubisco. El aumento en el contenido de azúcares solubles, principalmente sacarosa, ha sido observado en especies herbáceas y leñosas sometidas a tratamiento gradual en frío, asociado a una función crioprotectora y de ajuste osmótico para la protección contra el stress por enfriamiento y por déficit hídrico (Levitt 1980). Figuereido-Ribeiro y Dietrich (1983) trabajando con xilopodios de Ocimum nudicaule, una roseta herbácea pequeña, que crece en la sabana brasilera, observaron un aumento en el contenido de azúcares solubles, bajo un régimen de almacenamiento a $5-28{ }^{\circ} \mathrm{C}$ comparado con $5{ }^{\circ} \mathrm{C}$ constante. Estos autores concluyen en que se trata de una respuesta bioquímica rápida a las condiciones tropicales y subtropicales, donde las plantas pueden estar sujetas a variaciones cortas de temperaturas. Existen evidencias de aumento de temperatura interna foliar diurna en el frailejón debido al efecto de la incidencia de la radiación solar sobre la pubescencia y la geometría de la roseta (Larcher 1980, Meinzer y Goldstein 1985) lo cual contrastado con las bajas temperaturas nocturnas detectadas en el páramo y por extrapolación, resultaría comparable con la situación descrita por FiguereidoRibeiro y Dietrich (1983).

En referencia al contenido de proteínas, sí bien se observan diferencias, las mismas no son significativas y esto nos permite inferir que el aumento en la actividad Rubisco se debe a mayor activación de la enzima con la elevación; lo que a su vez soportaría los crecientes valores de azúcares solubles obtenidos con la altitud. En la relación entre el contenido de proteínas y la temperatura, existen evidencias contradictorias, ya que algunos estudios indican aumento a bajas temperaturas y otros no; Levitt (1980) 
explica estas contradicciones basándose en el método de extracción empleado.

$\mathrm{El}$ área foliar específica (AFE) disminuye a valores altos de radiación; este descenso es principalmente debido a la reducción del área foliar con la altitud y al aumento del peso seco. Torres (1980) encontró una reducción del tamaño celular y de los espacios intercelulares en las poblaciones de mayor elevación de E. schultzii, lo cual apoya los valores de AFE obtenidos en este trabajo. Adicionalmente, Milthorpe y Newton (1963) demostraron que a valores altos de radiación solar hay disminución del área foliar debido a reducción del tamaño celular, pero que el número de células aumenta hasta un valor constante. Körner (1989) afirma que la disminución de AFE es la tendencia morfológica más consistente en hojas a lo largo de un gradiente altitudinal; esta afirmación coincide con los resultados aquí presentados. Con la elevación ocurre un aumento por unidad de área de la actividad carboxilasa de la Rubisco y del contenido de azúcares solubles, lo cual indica una actividad metabólica superior soportada por el incremento en radiación solar y tasa fotosintética, para compensar el requerimiento de aumento de la pubescencia foliar como adaptación a condiciones extremas tales como excesivas radiaciones solar y ultravioleta, causantes de la fotooxidación del clorofila.

De los parámetros medidos en las tres poblaciones estudiadas, tres presentan diferencias significativas asociadas con la altitud: actividad Rubisco, contenido de azúcares solubles y AFE, mientras que los contenidos de clorofilas y proteínas no la muestran. Esto representa un equilibrio ("compromiso") entre una mayor actividad metabólica y un menor volumen celular.

\section{AGRADECIMIENTOS}

Agradezco al Decanato de Investigación y Desarrollo de la Universidad Simón Bolívar por el apoyo financiero, a O. Arenas por su asesoría en el tratamiento estadístico de los resultados y finalmente a Elena Ilarraza y David Querales por su ayuda en la extracción foliar.

\section{RESUMEN}

Se establece una comparación de algunos parámetros fotosintéticos: actividad Rubisco (Ribulosa 1, 5-bis-fosfato carboxilasa EC 4.1.1.39) contenidos de clorofilas, azúcares solubles totales y proteínas solubles totales y área foliar específica (AFE), en tres poblaciones altitudinales de Espeletia schultzii ubicadas a 3 100, 3550 y 4200 msnm. De acuerdo con el análisis de varianza la población a 4200 m presentó una actividad Rubisco significativamente mayor que las otras dos, en cuyos casos los valores no fueron diferentes. Los contenidos de clorofila a presentan una leve tendencia a la disminución con la altura; mientras que la clorofila b se mantiene constante, razón por la cual la relación a/b ( $\mathrm{R}$ a/b) disminuye con la elevación, aunque, las diferencias no son significativas. El contenido de azúcares en cada una de las poblaciones estudiadas $\left(\mathrm{gm}^{-2}\right)$, aumenta con la elevación; las diferencias entre las poblaciones son significativas. Con relación al contenido de proteínas, no existen diferencias entre las poblaciones estudiadas. El área foliar específica (AFE) disminuye significativamente con la altura. Es probable que el incremento en la actividad Rubisco sea producido por una mayor activación de la enzima y no por el mayor contenido de proteína. Los azúcares solubles presentan incremento significativo, este hecho es probable que se encuentre asociado con la actividad Rubisco. De los cinco parámetros fotosintéticos medidos, tres presentan diferencias significativas asociadas con la altitud. Esto representa un compromiso entre una mayor actividad metabólica mantenida en un menor volumen celular.

Palabras clave: clorofila, Espeletia, páramo, rubisco, proteínas solubles, azúcares solubles, área foliar específica.

\section{REFERENCIAS}

Baruch, Z. 1979. Elevational differentiation in Espeletia schultzii (Compositae), a giant rosette plants of the Venezuela paramos. Ecology 60: 85-98.

Baruch, Z. 1984. Ordination and Classification of vegetation along an altitudinal gradient in the Venezuela paramos. Vegetatio 5: 115-126.

Bradford, M.M. 1976. A rapid and sensitive method for the quantitation of microgram quantities of protein-dye utilizing the principle of protein dye binding. Anal. Biochem. 72: 248-254.

Bruinsma, J. 1963. The quantitative analysis of chlorophylss $\mathrm{a}$ and $\mathrm{b}$ in plant extracts. Photochem. Photobiol. 2: 241-249.

Castrillo, M. 1995. Ribulose-1, 5-bis-phosphate carboxylase activity in altitudinal populations of Espeletia schultzii Wedd. Oecologia 101: 193-196. 
Castrillo, M. \& M. Simoes. 1997. Leaf non-strutural carbohydrates and leaf dry weight per area in three altitudinal populations of Espeletia schultzii Wedd. Folia Geobot. Phytotax. 32: 355-360.

De los Ríos, C., D.H. Baez, Q. Contreras, O. Crescente \& A. Caserta. 1999. Phytochemical evaluation and antibacterial activity of Espeletia schlutzii (Asteraceae). Ciencia 1: 72-77.

Figuereido-Ribeiro, R.C.L. \& S.M.C. Dietrich. 1983. Sugar content and metabolic activities in cold -stored fragmented xylopodium of Ocimum nudicaule Benth var. anisifolia Giul (Labiateae). J. Exp. Bot. 34: 476-483.

Goldstein, G., F. Rada \& A. Azocar. 1985. Cold hardiness and supercooling along an altitudinal gradient in andean giant rosette. Oecologia 68: 147-152.

Kemp, P.R. \& G.J.III William. 1977. Temperature relations of gas exchange in altitudinal populations of Taraxacum officinale. Can J. Bot. 55: 2496-2502.

Khouri, N., A. Usubillaga, L.B. Rojas \& F. Galárraga. 2000. The essential oil of Espeletia weddeli Sch. Bip. Ex Wedd. Flavour Fragance J. 15: 263-265.

Körner, C.H. 1989. The nutritional status of plants from high altitudes. A worldwide comparison. Oecologia 81: 379-391.

Larcher, W. 1980. Physiological plant ecology. Springer, Berlín, Alemania. 303 p.

Levitt, J. 1980. Responses of plants to environmental stress Vol. II. Chilling, freezing and high temperature stresses Physiological Ecology Series. Academic, Nueva York, EEUU. 497 p.

Machler, F. \& J. Nosberger. 1977. Effect of light intensity and temperature on apparent photosynthesis of altitudinal ecotypes of Trifolium repens L. Oecologia 31: 73-78.

Meinzer, F. \& G. Goldstein. 1985. Some consequences of leaf pubescence in the andean giant rosette plant Espeletia timotensis. Ecology 66: 512-520.

Meinzer, F., G. Goldstein \& P. Rundell. 1985. Morphological changes and altitudinal gradient and their consequences for an Andean giant rosette plant. Oecologia 65: $278-283$

Milthorpe, F.L. \& D. Newton. 1963. Studies on the expansion of the leaf surface III. The influence of radiation on cell division and expansion. J. Exp. Bot. 14: 483-495.

Monasterio, M. 1980. Estudios ecológicos en los páramos andinos. Universidad de Los Andes, Mérida,Venezuela. 312 p.

Oulton, K., G.J.III Williams \& D.S. May. 1979. Ribulose1, 5-bis-phosphate carboxylase in Taraxacum officinale. Photosynthetica 13: 15-20.

Rada, F., J. Gonzalez, A. Azocar, B. Briceño \& R. Jaimez. 1992. Net photosynthesis-leaf temperature relations in plant species with different height along an altitudinal gradient. Acta Oecol. 13: 535-542.

Rada, F., A. Azocar, J. Gonzalez \& B. Briceño. 1998. Leaf gas exchange in Espeletia schultzii a giant caulescent rosette plant along an altitudinal gradient in the Venezuelan Andes. Acta Oecol. 19: 73-79.

Rauscher, J.T. 2002. Molecular phylogenetics of the Espeletia complex (Asteraceae): evidence from nrDNA ITS sequences on the closest relatives of an Andean adaptive radiation. Am. J. Bot. 89: 10741084 .

Silva, J., M.C. Trevisan, C.A. Estrada \& M. Monasterio. 2000. Comparative demography of two giant caulescent rosettes (Espeletia timotensis and E. spicata) from the high tropical Andes. Global Ecol. Biogeogr. 9: 403-411.

Sokal, R.R. \& F.J. Rohlf. 1969. Biometry. W.M. Freman, California, EEUU. 776 p.

Torres, F. 1980. Modificación de la anatomía foliar de Espeletia schultzii Wedd a lo largo de un gradiente altitudinal entre 2600 y $4200 \mathrm{msnm}$. In Memorias VIII Congreso Venezolano de Botánica, Venezuela. $170 \mathrm{p}$.

Tranquilini, W. 1979. Physiological ecology of the alpine timberline. Ecological studies 31. Springer, Berlin, Alemania. 137 p.

Usubillaga, A., N. Khouri, L.B. Rojas \& L. Morillo. 2001. Essential oil of the leaves from Espeletia batata Cuatrec. J. Essent. Oil Res. 13: 450-451.

Van Handel, E. 1968. Direct microdetermination of sucrose. Anal. Biochem. 22: 280-283.

Vareschi, V. 1970. La flora de los Páramos. Universidad de Los Andes, Mérida, Venezuela. 337 p. 
